\title{
AN ADDITIVE VERSION OF HIGHER CHOW GROUPS
}

\author{
By SPENCER BLOCH AND HÉLÈnE ESNAULT
}

Abstract. - The cosimplicial scheme

$$
\Delta^{\bullet}=\Delta^{0} \rightrightarrows \Delta^{1} \rightrightarrows \ldots ; \quad \Delta^{n}:=\operatorname{Spec}\left(k\left[t_{0}, \ldots, t_{n}\right] /\left(\sum t_{i}-t\right)\right)
$$

was used in (Bloch, S., Algebraic cycles and higher $K$-theory, Adv. Math. 61 (3) (1986) 267-304) to define higher Chow groups. In this note, we let $t$ tend to 0 and replace $\Delta^{\bullet}$ by a degenerate version

$$
Q^{\bullet}=Q^{0} \rightrightarrows Q^{1} \rightrightarrows \cdots ; \quad Q^{n}:=\operatorname{Spec}\left(k\left[t_{0}, \ldots, t_{n}\right] /\left(\sum t_{i}\right)\right)
$$

to define an additive version of the higher Chow groups. For a field $k$, we show the Chow group of 0 -cycles on $Q^{n}$ in this theory is isomorphic to the group of absolute $(n-1)$-Kähler forms $\Omega_{k}^{n-1}$.

An analogous degeneration on the level of de Rham cohomology associated to "constant modulus" degenerations of varieties in various contexts is discussed.

๑) 2003 Éditions scientifiques et médicales Elsevier SAS

RÉSUMÉ. - Le schéma cosimplicial

$$
\Delta^{\bullet}=\Delta^{0} \rightrightarrows \Delta^{1} \rightrightarrows \ldots ; \quad \Delta^{n}:=\operatorname{Spec}\left(k\left[t_{0}, \ldots, t_{n}\right] /\left(\sum t_{i}-t\right)\right)
$$

a été utilisé dans (Bloch, S., Algebraic cycles and higher $K$-theory, Adv. Math. 61 (3) (1986) 267-304) afin de définir des groupes de Chow supérieurs. Dans cette note, nous définissons une version additive des groupes de Chow supérieurs en faisant tendre $t$ vers 0 et en remplaçant $\Delta^{\bullet}$ par une version dégénérée

$$
Q^{\bullet}=Q^{0} \rightrightarrows Q^{1} \rightrightarrows \ldots ; \quad Q^{n}:=\operatorname{Spec}\left(k\left[t_{0}, \ldots, t_{n}\right] /\left(\sum t_{i}\right)\right)
$$

Nous montrons que sur un corps $k$, le groupe de Chow des 0-cycles dans cette théorie est isomorphe aux formes de Kähler absolues de degré $(n-1)$.

Nous discutons une dégénerescence analogue en cohomologie de de Rham apparaissant dans diverses situations pour des familles à module constant.

(ㄷ) 2003 Éditions scientifiques et médicales Elsevier SAS

\section{Introduction}

The purpose of this note is to study a common sort of limiting phenomenon which occurs in the study of motives. Here is a simple example. Let $k$ be a field. Let $S=\mathbb{A}_{t}^{1}=\operatorname{Spec}(k[t])$ 
and let $T=\operatorname{Spec}\left(k[x, t] /(x(x-t)) \hookrightarrow \mathbb{A}_{t, x}^{2}\right.$. Over $S[1 / t]$ the Picard scheme $\operatorname{Pic}\left(\mathbb{A}_{x, t}^{2}, T\right) / S$ is represented by $\mathbb{G}_{m, S[1 / t]}$. On the other hand, when $t=0$ one gets $\operatorname{Pic}\left(\mathbb{A}_{x}^{1},\left\{x^{2}=0\right\}\right) \cong \mathbb{G}_{a, k}$. In some sense, $\mathbb{G}_{m}$ has "jumped" to $\mathbb{G}_{a}$.

In higher dimension, for $t \neq 0$, the homology of the group of algebraic cycles associated to the cosimplicial scheme

$$
\Delta^{\bullet}=\Delta^{0} \rightrightarrows \Delta^{1} \rightrightarrows \ldots ; \quad \Delta^{n}:=\operatorname{Spec}\left(k\left[t_{0}, \ldots, t_{n}\right] /\left(\sum t_{i}-t\right)\right)
$$

is known to give motivic cohomology [9]. What can one say about the algebraic cycle groups (1.3) of the degenerate cosimplicial complex:

$$
Q^{\bullet}=Q^{0} \rightrightarrows Q^{1} \rightrightarrows \ldots ; \quad Q^{n}:=\operatorname{Spec}\left(k\left[t_{0}, \ldots, t_{n}\right] /\left(\sum t_{i}\right)\right) ?
$$

Our main result is a calculation of the Chow groups of 0 -cycles on $Q^{\bullet}$. Let $\mathcal{Z}^{n}\left(Q^{r}\right)$ be the free abelian group on codimension $n$ algebraic cycles on $Q^{r}$ satisfying a suitable general position condition with respect to the face maps. Let $S H^{n}(k, r)$ be the cohomology groups of the complex

$$
\cdots \rightarrow \mathcal{Z}^{n}\left(Q^{r+1}\right) \rightarrow \mathcal{Z}^{n}\left(Q^{r}\right) \rightarrow \mathcal{Z}^{n}\left(Q^{r-1}\right) \rightarrow \cdots
$$

where the boundary maps are alternating sums of pullbacks along face maps. Write $\Omega_{k}^{\bullet}$ for the absolute Kähler differentials.

THEOREM 1.1.-

$$
S H^{n}(k, n) \cong \Omega_{k}^{n-1} .
$$

Note for $n=1$, this is the above $\mathbb{G}_{a}$.

We explain another example of this limiting phenomenon. We consider $\mathbb{P}^{n+1}$ with homogeneous coordinates $U_{0}, \ldots, U_{n+1}$ over a field $k$. Let

$$
X: f\left(U_{0}, \ldots, U_{n+1}\right)=0
$$

be a hypersurface $X$ defined by a homogeneous polynomial $f$ of degree $n+2$ (e.g. an elliptic curve in $\mathbb{P}^{2}$ ).

Write $u_{i}=U_{i} / U_{0}$, and define a $\log (n+1)$-form on $\mathbb{P}^{n+1} \backslash X$

$$
\omega_{f}:=\frac{d u_{1} \wedge \cdots \wedge d u_{n+1}}{f\left(1, u_{1}, \ldots, u_{n+1}\right)} .
$$

As well known, this form generates $\omega_{\mathbb{P}^{n+1}}(X) \cong \mathcal{O}_{\mathbb{P}^{n+1}}$.

Let $r_{1}, \ldots, r_{n+1} \geqslant 0$ be integers. We consider the action of $\mathbb{G}_{m}$ given by the substitutions $u_{i}=t^{-r_{i}} v_{i}$. Let $N$ be minimal such that $t^{N} f\left(1, t^{-r_{1}} u_{1}, \ldots, t^{-r_{n+1}} u_{n+1}\right)$ is integral in $t$. Assume $s:=N-\sum r_{i}>0$ and $s$ is invertible in $k$. One checks easily that in the coordinates $v_{i}$ one has

$$
\omega_{f}=t^{s} \nu_{f}\left(t, v_{1}, \ldots, v_{n+1}\right)+s t^{s-1} d t \wedge \gamma_{f}\left(t, v_{1}, \ldots, v_{n+1}\right)
$$

with 


$$
\begin{gathered}
\nu_{f}\left(t, v_{1}, \ldots, v_{n+1}\right)=\frac{d v_{1} \wedge \cdots \wedge d v_{n+1}}{t^{N} f\left(1, t^{-r_{1}} v_{1}, \ldots, t^{-r_{n+1}} v_{n+1}\right)} \\
\gamma_{f}\left(t, v_{1}, \ldots, v_{n+1}\right)=\frac{1}{s} \sum_{i}(-1)^{i} r_{i} v_{i} \frac{d v_{1} \wedge \cdots \wedge \widehat{d v_{i}} \wedge \cdots \wedge d v_{n+1}}{t^{N} f\left(1, t^{-r_{1}} v_{1}, \ldots, t^{-r_{n+1}} v_{n+1}\right)} .
\end{gathered}
$$

Thus $\nu_{f}$ and $\gamma_{f}$ are forms in $d v_{i}$ of degrees $n+1$ and $n$ respectively which are integral in $t$. As $\omega_{f}$ is an $(n+1)$-form on $\mathbb{P}^{n+1}$ in the $u$-coordinates, it is closed, so

$$
s t^{s-1} d t \wedge\left(\nu_{f}\left(t, v_{1}, \ldots, v_{n+1}\right)-d \gamma_{f}\left(t, v_{1}, \ldots, v_{n+1}\right)\right)+s t^{s-1} \cdot \frac{t}{s} d \nu_{f}\left(t, v_{1}, \ldots, v_{n+1}\right)=0 .
$$

Dividing by $t^{s-1}$ and restricting to $t=0$ yields

$$
\left.\nu_{f}\right|_{t=0}=\left.d \gamma_{f}\right|_{t=0} .
$$

In other words, under the action of the 1-parameter subgroup, $\nu_{f}$ degenerates to the exact form $d \gamma_{f}$.

To see the relationship with the 0 -cycles, take

$$
\Delta_{n+1}: f\left(1, u_{1}, \ldots, u_{n+1}\right)=u_{1} u_{2} \cdots u_{n+1}\left(1-u_{1}-\cdots-u_{n+1}\right)=0 .
$$

Substitute $v_{i}=t u_{i}, 1 \leqslant i \leqslant n+1$. Then $s=1$, and a calculation yields

$$
\begin{gathered}
\gamma_{\Delta_{n+1}}\left(:=\gamma_{f}\right)=\frac{\left.\sum_{i=1}^{n+1}(-1)^{i} d \log \left(v_{1}\right) \wedge \cdots \wedge d \widehat{\log \left(v_{i}\right.}\right) \wedge \cdots \wedge d \log \left(v_{n+1}\right)}{t-v_{1}-\cdots-v_{n+1}} \\
\nu_{\Delta_{n+1}}\left(:=\nu_{f}\right)=\frac{d v_{1} \wedge \cdots \wedge d v_{n+1}}{v_{1} v_{2} \cdots v_{n+1}\left(t-v_{1}-\cdots-v_{n+1}\right)}
\end{gathered}
$$

Note the limiting configuration as $t \rightarrow 0$ is (compare (1.2))

$$
v_{1} v_{2} \cdots v_{n+1}\left(v_{1}+\cdots+v_{n+1}\right)=0 .
$$

For convenience we write

$$
\gamma_{n}:=\left.\gamma_{\Delta_{n+1}}\right|_{t=0}
$$

We view $\left.\nu_{\Delta_{n+1}}\right|_{t=1}$ (resp. $\gamma_{n}$ ) as a map

$$
\mathcal{Z}_{0}\left(\mathbb{A}_{k}^{n+1} \backslash\left\{\left(1-u_{1}+\cdots+u_{n+1}\right) u_{1} \cdots u_{n+1}=0\right\}\right) \rightarrow \Omega_{k}^{n+1}
$$

(resp.

$$
\left.\mathcal{Z}_{0}\left(\mathbb{A}_{k}^{n+1} \backslash\left\{v_{1} v_{2} \cdots v_{n+1}\left(v_{1}+\cdots+v_{n+1}\right)=0\right\}\right) \rightarrow \Omega_{k}^{n}\right) .
$$

Here $\mathcal{Z}_{0}$ denotes the free abelian group on closed points (0-cycles) and the maps are respectively

$$
\left.x \mapsto \operatorname{Tr}_{k(x) / k} \nu\right|_{\{x\}} ;\left.\quad x \mapsto \operatorname{Tr}_{k(x) / k} \gamma\right|_{\{x\}} .
$$

In the first case, the Nesterenko-Suslin-Totaro theorem [7,8] identifies the zero cycles modulo relations coming from curves in $\mathbb{A}^{n+2}$ with the Milnor $K$-group $K_{n+1}^{M}(k)$. The evaluation map 
(1.10) passes to the quotient, and the resulting map $K_{n+1}^{M}(k) \rightarrow \Omega_{k}^{n+1}$ is given on symbols by the $d \log$-map

$$
\left\{x_{1}, \ldots, x_{n+1}\right\} \mapsto d \log \left(x_{1}\right) \wedge \cdots \wedge d \log \left(x_{n+1}\right) .
$$

In the second case, factoring out by the relations coming from curves on $\mathbb{A}^{n+2}$ as in (1.3) yields the Chow group of 0 -cycles $S H^{n+1}(k, n+1)$, and our main result is that evaluation on $\gamma_{n}$ gives an isomorphism

$$
S H^{n+1}(k, n+1) \cong \Omega_{k}^{n} .
$$

Sections 2-5 contain the proof of Theorem 1.1. Section 6 contains some brief remarks on specialization of forms as it relates to Aomoto's theory of configurations, to 0-cycles on hypersurfaces, and to Goncharov's theory of hyperbolic motives. Finally, Section 7 computes $S H^{1}(k, n)$, responding to a question of $\mathrm{S}$. Lichtenbaum.

\section{The additive Chow groups}

In this section, we consider a field $k$, and a $k$-scheme $X$ of finite type.

We will throughout use the following notations.

Notations 2.1. - We set $Q^{n}=\operatorname{Spec} k\left[t_{0}, \ldots, t_{n}\right] /\left(\sum_{i=0}^{n} t_{i}\right)$, together with the faces

$$
\partial_{j}: Q^{n-1} \rightarrow Q^{n} ; \quad \partial_{j}^{*}\left(t_{i}\right)= \begin{cases}t_{i} & i<j, \\ 0 & i=j, \\ t_{i-1} & i>j .\end{cases}
$$

One also has degeneracies

$$
\pi_{j}: Q^{n} \rightarrow Q^{n-1} ; \quad \pi_{j}^{*}\left(t_{i}\right)= \begin{cases}t_{i} & i<j \\ t_{i}+t_{i+1} & i=j \\ t_{i+1} & i>j\end{cases}
$$

We denote by $\{0\} \in Q^{n}$ the vertex defined by $t_{i}=0$. We write $Q_{X}^{n}=Q^{n} \times_{\operatorname{Spec}(k)} X$. The above face and degeneracy maps make $Q_{X}^{\bullet}$ a cosimplicial scheme.

DEFINITION 2.2. - Let $\mathcal{S Z}_{q}(X, n)$ be the free abelian group on irreducible, dimension $q$ subvarieties in $Q_{X}^{n}$ with the property:

(i) They don't meet $\{0\} \times X$.

(ii) They meet all the faces properly, that is in dimension $\leqslant q$.

Thus the face maps induce restriction maps

$$
\partial_{i}: \mathcal{S Z}_{q}(X, n) \rightarrow \mathcal{S Z}_{q-1}(X, n-1) ; \quad i=0, \ldots, n ; \partial=\sum_{i=0}^{n}(-1)^{i} \partial_{i}
$$

yielding complexes $\mathcal{S Z}_{q-\bullet}(X, \bullet)$ :

$$
\cdots \stackrel{\partial}{\rightarrow} \mathcal{S Z}_{q+1}(X, n+1) \stackrel{\partial}{\rightarrow} \mathcal{S Z}_{q}(X, n) \stackrel{\partial}{\rightarrow} \mathcal{S Z}_{q-1}(X, n-1) \stackrel{\partial}{\rightarrow} \cdots
$$


DEFINITION 2.3. - The additive higher Chow groups are given for $n \geqslant 1$ by

$$
S H_{q}(X, n)=H_{n}\left(\mathcal{S Z}_{q-\bullet}(X, \bullet)\right)
$$

and (for $X$ equidimensional)

$$
S H^{p}(X, n)=S H_{\operatorname{dim} \mathrm{X}-p}(X, n) .
$$

The groups are not defined for $n=0$.

Remarks 2.4.-

(i) The above should be compared with the higher Chow groups $C H^{p}(X, n)$ defined as above with $Q^{\bullet}$ replaced by $\Delta^{\bullet}$, where $\Delta^{n}:=\operatorname{Spec}\left(k\left[t_{0}, \ldots, t_{n}\right] /\left(\sum t_{i}-1\right)\right)$.

(ii) The cosimplicial scheme $Q^{\bullet}$ admits an action of $\mathbb{G}_{m, k}$, which we define by

$$
x \star\left(t_{0}, \ldots, t_{n}\right):=\left(t_{0} / x, \ldots, t_{n} / x\right) .
$$

(The reason for the inverse will be clear below.) By functoriality, we obtain a $k^{\times}$-action on the $S H^{p}(X, n)$.

(iii) Let $f: X^{\prime} \rightarrow X$ be a proper map with $n=\operatorname{dim} X^{\prime}-\operatorname{dim} X$. Then one has a push-forward map

$$
f_{*}: \mathcal{S Z ^ { p }}\left(X^{\prime}, \bullet\right) \rightarrow \mathcal{S Z ^ { p - n }}(X, \bullet) .
$$

On homology this yields $S H^{p}\left(X^{\prime}, n\right) \rightarrow S H^{p-n}(X, n)$. We will be particularly interested in the case $X=\operatorname{Spec}(k), X^{\prime}=\operatorname{Spec}\left(k^{\prime}\right)$ with $\left[k^{\prime}: k\right]<\infty$. We write $\operatorname{tr}_{k^{\prime} / k}: S H^{p}\left(k^{\prime}, n\right) \rightarrow S H^{p}(k, n)$ for the resulting map. This trace map is compatible with the action of $k^{\times}$from (ii) in the sense that for $x \in k^{\times}$and $a^{\prime} \in S H^{p}\left(k^{\prime}, n\right)$ we have $x \star \operatorname{tr}_{k^{\prime} / k}\left(a^{\prime}\right)=\operatorname{tr}_{k^{\prime} / k}\left(x \star a^{\prime}\right)$.

LEMMA 2.5. - The $\star$ action of $k^{\times}$on $S H^{n}(k, n)$ extends to an action of the multiplicative monoid $k$ by setting $0 \star x=0$. This action comes from a $k$-vector space structure on $S^{n}(k, n)$.

Proof. - We have to show that for a closed point $x=\left(u_{0}, \ldots, u_{n}\right) \in Q^{n} \backslash \bigcup_{i=0}^{n} \partial_{i}\left(Q^{n-1}\right)$, and $a, b \in k$, one has $(a+b) \star x=a \star x+b \star x$. For either $a$ or $b=0$, this is trivial. Thus we assume $a b \neq 0$. Let $k^{\prime}=k(x)$. Then the class in $S H^{n}(k, n)$ of $x$ is the trace from $k^{\prime}$ to $k$ of a $k^{\prime}$-rational point $x^{\prime} \in S H^{n}\left(k^{\prime}, n\right)$. Using the compatibility of $\star$ and trace from Remarks 2.4(iii) above, we reduce to the case $x k$-rational.

Write $\left(t_{0}-u_{0}, \ldots, t_{n}-u_{n}\right)$ for the ideal of $x$. Define $\ell(t)=-\frac{a b}{u_{0}} t+a+b$. Consider the curve $W \subset Q^{n+1}$ defined parametrically by

$$
W=\left\{\left(t,-t+\frac{u_{0}}{\ell(t)}, \frac{u_{1}}{\ell(t)}, \ldots, \frac{u_{n}}{\ell(t)}\right)\right\} .
$$

To check that this parametrized locus is Zariski-closed, we consider the ideal:

$$
I_{W}=\left(\left(t_{1}+t_{0}\right) \ell\left(t_{0}\right)-u_{0}, t_{2} \ell\left(t_{0}\right)-u_{1}, \ldots, t_{n} \ell\left(t_{0}\right)-u_{n}\right) .
$$

If $y=\left(y_{0}, \ldots, y_{n+1}\right)$ is a geometric point in the zero locus of $I_{W}$, then since the $u_{i} \neq 0$ we see that $\ell\left(y_{0}\right) \neq 0$. Substituting $t=y_{0}$, we see that $y$ lies on the parametrized locus $W$.

The equation $-t+\frac{u_{0}}{\ell(t)}=0$ leads to a quadratic equation in $t$ with solutions $t=\frac{u_{0}}{a}, t=\frac{u_{0}}{b}$. If $a+b \neq 0$ we have 


$$
\begin{aligned}
& \partial_{0}(W)=\left(\frac{u_{0}}{a+b}, \ldots, \frac{u_{n}}{a+b}\right)=(a+b) \star\left(u_{0}, \ldots, u_{n}\right), \\
& \partial_{1}(W)=\left(\frac{u_{0}}{a}, \ldots, \frac{u_{n}}{a}\right)+\left(\frac{u_{0}}{b}, \ldots, \frac{u_{n}}{b}\right)=a \star\left(u_{0}, \ldots, u_{n}\right)+b \star\left(u_{0}, \ldots, u_{n}\right), \\
& \partial_{i}(W)=0, \quad i \geqslant 2,
\end{aligned}
$$

so the lemma follows in this case. If $a+b=0$, then $\partial_{0} W=0$ as well, and again the assertion is clear.

\section{Additive Chow groups and Milnor $K$-theory}

We consider the map (compatible with faces) $\iota: \Delta^{\bullet} \rightarrow Q^{\bullet+1}$ defined on $k$-rational points by $\left(u_{0}, \ldots, u_{n}\right) \mapsto\left(-1, u_{0}, \ldots, u_{n}\right)$. It induces a map of complexes $\mathcal{Z}^{p}(k, \bullet) \rightarrow \mathcal{S Z}^{p+1}(k, \bullet+1)$, which in turn induces a map

$$
\iota: C H^{p}(k, n) \rightarrow S H^{p+1}(k, n+1) .
$$

By [7] and [8], one has an isomorphism

$$
K_{n}^{M}(k) \cong C H^{n}(k, n)
$$

of the higher Chow groups of 0 -cycles with Milnor $K$-theory. It is defined by:

$$
\begin{gathered}
\left(u_{0}, \ldots, u_{n}\right) \mapsto\left\{-\frac{u_{0}}{u_{n}}, \ldots,-\frac{u_{n-1}}{u_{n}}\right\}, \\
\left\{b_{1}, \ldots, b_{n}\right\} \mapsto\left(\frac{b_{1}}{c}, \ldots, \frac{b_{n}}{c},-\frac{1}{c}\right) ; \quad c=-1+\sum_{i=1}^{n} b_{i} .
\end{gathered}
$$

Note that if $\sum_{i=1}^{n} b_{i}=1$, then the symbol $\beta:=\left\{b_{1}, \ldots, b_{n}\right\}$ is trivial in Milnor $K$-theory, and one maps $\beta$ to 0 .

In this way, one obtains a map

$$
\begin{aligned}
& K_{n-1}^{M}(k) \rightarrow S H^{n}(k, n) ; \\
& \quad\left\{x_{1}, \ldots, x_{n-1}\right\} \mapsto\left(-1, \frac{x_{1}}{-1+\sum_{i=1}^{n-1} x_{i}}, \ldots, \frac{x_{n-1}}{-1+\sum_{i=1}^{n-1} x_{i}}, \frac{-1}{-1+\sum_{i=1}^{n-1} x_{i}}\right) .
\end{aligned}
$$

\section{Differential forms}

In this section we construct a $k$-linear map $\Omega_{k}^{n-1} \rightarrow S H^{n}(k, n)$. (Here $\Omega_{k}^{i}$ are the absolute Kähler differential $i$-forms.)

The following lemma is closely related to calculations in [5].

LEMMA 4.1.- As a k-vector space, the differential forms $\Omega_{k}^{n-1}$ are isomorphic to $\left(k \otimes_{\mathbb{Z}} \wedge^{n-1} k^{\times}\right) / \mathcal{R}$. The $k$-structure on $k \otimes_{\mathbb{Z}} \wedge^{n-1} k^{\times}$is via multiplication on the first argument. The relations $\mathcal{R}$, for $n \geqslant 2$, are the $k$-subspace spanned by

$$
a \otimes\left(a \wedge b_{1} \wedge \cdots \wedge b_{n-2}\right)+(1-a) \otimes\left((1-a) \wedge b_{1} \wedge \cdots \wedge b_{n-2}\right),
$$


for $b_{i} \in k^{\times}, a \in k$. The map $\left(k \otimes_{\mathbb{Z}} \wedge^{n-1} k^{\times}\right) / \mathcal{R} \rightarrow \Omega_{k}^{n-1}$ is then defined by

$$
\left(a, b_{1}, \ldots, b_{n-1}\right) \mapsto a d \log b_{1} \wedge \cdots \wedge d \log b_{n-1} .
$$

Proof. - Write ' $\Omega^{*}:=\left(k \otimes_{\mathbb{Z}} \wedge^{*} k^{\times}\right) / \mathcal{R}$. This is a quotient of the graded $k$-algebra $k \otimes_{\mathbb{Z}} \wedge^{*} k^{\times}$ by the graded ideal $\mathcal{R}$ and hence has a graded $k$-algebra structure, generated in degree 1 . There is an evident surjection of graded algebras ' $\Omega^{*} \rightarrow \Omega^{*}$, so, by the universal mapping property of the exterior algebra $\Omega^{*}$, it suffices to check' $\Omega^{1} \cong \Omega^{1}$.

Define $D: k \rightarrow\left(k \otimes_{\mathbb{Z}} k^{\times}\right) / \mathcal{R}$ by $D(a)=a \otimes a$ for $a \in k^{\times}$, else $D(0)=0$. To define the required inverse, it suffices to show that $D$ is a derivation. Clearly, $D(a b)=a D(b)+b D(a)$. Also $1 \otimes-1$ is trivial in $k \otimes k^{\times}$, so $D(-a)=-D(a)$. Given $a, b \in k^{\times}$, write $b=-a c$. We have

$$
\begin{aligned}
& D(a+b)=D(a-a c)=a D(1-c)+(1-c) D(a) \\
& \quad=-a D(c)+D(a)-c D(a)=D(a)+D(-a c)=D(a)+D(b) .
\end{aligned}
$$

Hence $D$ is a derivation so the inverse map $\Omega^{1} \rightarrow{ }^{\prime} \Omega^{1}$ is defined.

Remark 4.2. - We will frequently use the relations in the equivalent form

$$
a \otimes a \wedge(\cdots)-(1+a) \otimes(1+a) \wedge(\cdots) \sim 0 .
$$

Proposition 4.3. - One has a well-defined k-linear map

$$
\begin{aligned}
\phi: \Omega_{k}^{n-1} & \rightarrow S H^{n}(k, n), \\
\alpha:=a d \log b_{1} \wedge \cdots \wedge d \log b_{n-1} & \mapsto a \star\left(-1, \frac{b_{1}}{\gamma}, \ldots, \frac{b_{n-1}}{\gamma},-\frac{1}{\gamma}\right),
\end{aligned}
$$

where $\gamma=-1+\sum_{i=1}^{n-1} b_{i}$. (Define $\alpha=0$ when $\gamma=0$.) The diagram

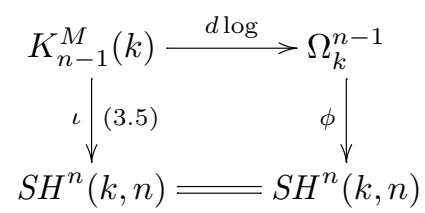

is commutative.

Proof. - We write $c=-1+a+\sum_{i=2}^{n-1} b_{i}$. By Lemma 4.1, we have to show

$$
0=\rho:=a \star\left(-1, \frac{a}{c}, \ldots, \frac{b_{n-1}}{c},-\frac{1}{c}\right)-(a+1) \star\left(-1, \frac{a+1}{c+1}, \ldots, \frac{b_{n-1}}{c+1},-\frac{1}{c+1}\right) .
$$

If $a=0$, then one has

$$
\rho=-\left(-1, \frac{1}{c}, \ldots, \frac{b_{n-1}}{c},-\frac{1}{c}\right)=-\iota\left\{1, b_{2}, \ldots, b_{n-1}\right\}=0 .
$$

Similarly, $\rho=0$ if $a=-1$. Assume now $a \neq 0,-1$. Set $b=-a \in k \backslash\{0,1\}$. One defines, for $n \geqslant 3$ and $\left(u_{1}, \ldots, u_{n-1}\right) \in\left(\Delta^{n-2} \backslash \bigcup_{i=1}^{n-1} \Delta^{n-3}\right)(k)$, the parametrized curve

$$
\Gamma(b, u):=\left\{\left(\frac{-1}{b}+t, \frac{1}{b-1},-t, \frac{-u_{1}}{b(b-1)}, \ldots, \frac{-u_{n-1}}{b(b-1)}\right)\right\} \subset Q^{n+1}
$$


and for $n=2$

$$
\Gamma(b):=\left\{\left(\frac{-1}{b}+t, \frac{1}{b-1},-t, \frac{-1}{b(b-1)}\right)\right\} \subset Q^{3} .
$$

(See [8] for the origin of this definition.) This curve is indeed in good position, so it lies in $\mathcal{S Z}^{n}\left(Q^{n+1}\right)$. One computes

$$
\begin{aligned}
\partial \Gamma(b, u)= & (1-b) \star\left(-1,1-\frac{1}{b}, \frac{u_{1}}{b}, \ldots, \frac{u_{n-1}}{b}\right) \\
& +b \star\left(-1, \frac{b}{b-1}, \frac{-u_{1}}{b-1}, \ldots, \frac{-u_{n-1}}{b-1}\right) .
\end{aligned}
$$

(Resp. in the case $n=2$

$$
\left.\partial \Gamma(b)=(1-b) \star\left(-1,1-\frac{1}{b}, \frac{1}{b}\right)+b \star\left(-1, \frac{b}{b-1}, \frac{-1}{b-1}\right) .\right)
$$

Now one has

$$
\begin{aligned}
& \left(-1,1-\frac{1}{b}, \frac{u_{1}}{b}, \ldots, \frac{u_{n-1}}{b}\right) \\
& =\iota\left\{\frac{1-b}{u_{n-1}},-\frac{u_{1}}{u_{n-1}}, \ldots,-\frac{u_{n-2}}{u_{n-1}}\right\} \\
& =\iota\left[\left\{1-b,-\frac{u_{1}}{u_{n-1}}, \ldots,-\frac{u_{n-2}}{u_{n-1}}\right\}-\left\{u_{n-1}, u_{1}, \ldots, u_{n-2}\right\}\right],
\end{aligned}
$$

as the rest of the multilinear expansion contains only symbols of the shape $\left\{\ldots, u_{n-1}, \ldots\right.$, $\left.-u_{n-1}, \ldots\right\}$. On the other hand, since $\sum_{i=1}^{n-1} u_{i}=1$, one has $\left\{u_{n-1}, u_{1}, \ldots, u_{n-2}\right\}=0$. Similarly, one has

$$
\left(-1, \frac{b}{b-1}, \frac{-u_{1}}{b-1}, \ldots, \frac{-u_{n-1}}{b-1}\right)=\iota\left\{\frac{b}{u_{n-1}},-\frac{u_{1}}{u_{n-1}}, \ldots,-\frac{u_{n-2}}{u_{n-1}}\right\} .
$$

The same argument yields that this is

$$
\iota\left\{b,-\frac{u_{1}}{u_{n-1}}, \ldots,-\frac{u_{n-2}}{u_{n-1}}\right\} .
$$

It follows now from (4.3) that for $n \geqslant 3$ we have the relation in $S H^{n}(k, n)$

$$
(1-b) \star \iota\left\{1-b,-\frac{u_{1}}{u_{n-1}}, \ldots,-\frac{u_{n-2}}{u_{n-1}}\right\}+b \star \iota\left\{b,-\frac{u_{1}}{u_{n-1}}, \ldots,-\frac{u_{n-2}}{u_{n-1}}\right\}=0
$$

(The analogous relation for $n=2$ is similar.)

Proposition 4.4. - With notation as above, the map

$$
\phi: \Omega_{k}^{n-1} \rightarrow S H^{n}(k, n)
$$

is surjective. In particular, $S H^{n}(k, n)$ is generated by the classes of $k$-rational points in $Q^{n}$. 
Proof. - It is easy to check that the image of $\phi$ coincides with the subgroup of $S H^{n}(k, n)$ generated by $k$-points. Clearly, $S H^{n}(k, n)$ is generated by closed points, and any closed point is the trace of a $k^{\prime}$-rational point for some finite extension $k^{\prime} / k$. We first reduce to the case $k^{\prime} / k$ separable. If $x \in Q_{k}^{n}$ is a closed point in good position (i.e. not lying on any face) such that $k(x) / k$ is not separable, then a simple Bertini argument shows there exists a curve $C$ in good position on $Q^{n+1}$ such that $\partial C=x+y$ where $y$ is a zero cycle supported on points with separable residue field extensions over $k$. Indeed, let $W \subset Q^{n+1}$ be the union of the faces. View $x \in W$. Since $x$ is in good position, it is a smooth point of $W$. Bertini will say that a nonempty open set in the parameter space of $n$-fold intersections of hypersurfaces of large degree containing $x$ will meet $W$ in $x$ plus a smooth residual scheme. Since $k$ is necessarily infinite, there will be such an $n$-fold intersection defined over $k$. Since the residual scheme is smooth, it cannot contain inseparable points. Then $x \equiv-y$ which is supported on separable points.

We assume now $k^{\prime} / k$ finite separable, and we must show that the trace of a $k^{\prime}$-point is equivalent to a zero cycle supported on $k$-points. Since the image of $\phi$ is precisely the subgroup generated by $k$-rational points, it suffices to check that the diagram

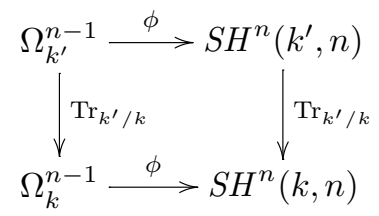

commutes. Because $k^{\prime} / k$ is separable, one has $\Omega_{k}^{n-1} \hookrightarrow \Omega_{k^{\prime}}^{n-1}$, and $\Omega_{k^{\prime}}^{n-1}=k^{\prime} \cdot \Omega_{k}^{n-1}$. One reduces to showing, for $\alpha=\left(-1, \alpha_{1}, \ldots, \alpha_{n}\right) \in Q^{n}(k)$ and $t \in k^{\prime}$, that $\operatorname{Tr}(t \star \alpha)=(\operatorname{Tr}(t)) \star \alpha$ in $S H^{n}(k, n)$.

Let $P(V)=V^{N}+a_{N-1} V^{N-1}+\cdots+a_{1} V+a_{0} \in k[V]$ be the minimal polynomial of $-\frac{1}{t}$. We set $b_{N}=\frac{-1}{\alpha_{n}}, b_{i}=\frac{-a_{i}}{\alpha_{n}}, i=N-1, \ldots, 2$ and $b_{i}=a_{i}, i=1,0$. We define the polynomial $Q(V, u)=b_{N} V^{N-1} u+\cdots+b_{2} V u+b_{1} V+b_{0} \in k[V, u]$, which by definition fulfills $Q\left(V,-\alpha_{n} V\right)=P(V)$. We define the ideal

$$
\mathcal{I}=\left(Q\left(V_{0}, u\right), V_{1}+\alpha_{1} V_{0}, \ldots, V_{n-1}+\alpha_{n-1} V_{0}\right) \subset k\left[V_{0}, \ldots, V_{n-1}, u\right] .
$$

It defines a curve $W \subset \mathbb{A}^{n+1}$. We think of $\mathbb{A}^{n+1}$ as being $Q^{n+1}$ with the faces $V_{0}=0$, $\ldots, V_{n-1}=0, u=0, u+\sum_{i=0}^{n-1} V_{i}=0$. Then this curve is in general position and defines a cycle in $\mathcal{S Z}^{1}(k, n+1)$.

Since $b_{0} \neq 0$, and $\alpha_{i} \neq 0$, one has

$$
\partial_{i} W=0, \quad i=0,1, \ldots, n-1
$$

One has

$$
\partial_{u} W \text { defined by }\left(a_{1} V_{0}+a_{0}, V_{1}+\alpha_{1} V_{0}, \ldots, V_{n-1}+\alpha_{n-1} V_{0}\right) .
$$

To compute the last face, we observe that the ideal

$$
\left(u+\sum_{i=0}^{n-1} V_{i}, V_{1}+\alpha_{1} V_{0}, \ldots, V_{n-1}+\alpha_{n-1} V_{0}\right),
$$


contains $u+\alpha_{n} V_{0}$. Consequently $\partial_{u+\sum_{i=0}^{n-1} V_{i}} W$ is defined by

$$
\left(Q\left(V_{0},-\alpha_{n} V_{0}\right), V_{1}+\alpha_{1} V_{0}, \ldots, V_{n-1}+\alpha_{n-1} V_{0}\right),
$$

with $Q\left(V_{0},-\alpha_{n} V_{0}\right)=P\left(V_{0}\right)$. Thus one obtains

$$
0 \equiv(-1)^{n} \partial W=\frac{a_{1}}{a_{0}} \star(-1, \alpha)-t \star(-1, \alpha) .
$$

Since $P$ is the minimal polynomial of $-\frac{1}{t}, \frac{a_{1}}{a_{0}}$ is the trace of $t$.

\section{The main theorem}

Recall (1.8) we have a logarithmic $(n-1)$-form $\gamma_{n-1}$ on $Q^{n}=\operatorname{Spec}\left(k\left[v_{0}, \ldots, v_{n}\right] /\left(\sum_{i=0}^{n} v_{i}\right)\right)$

$$
\begin{gathered}
\left.\gamma_{n-1}=\frac{1}{v_{0}} \sum_{1}^{n}(-1)^{i} d \log \left(v_{1}\right) \wedge \cdots \wedge d \widehat{\log \left(v_{i}\right.}\right) \wedge \cdots \wedge d \log \left(v_{n}\right), \\
d \gamma_{n-1}=\nu_{n}=\frac{d v_{1} \wedge \cdots \wedge d v_{n}}{v_{0} v_{1} \cdots v_{n}}
\end{gathered}
$$

Writing $v_{i}=V_{i} / V_{n+1}$, we can view $\gamma_{n-1}$ as a meromorphic form on

$$
\mathbb{P}^{n}=\operatorname{Proj}\left(k\left[V_{0}, \ldots, V_{n+1}\right] /\left(\sum_{0}^{n} V_{i}\right)\right) .
$$

Let $\mathcal{A}: V_{0} \cdots V_{n}=0 ; \infty: V_{n+1}=0$. The fact that $d \gamma_{n-1}$ has log poles on the divisors $V_{i}=0,0 \leqslant i \leqslant n$ implies that

$$
\gamma_{n-1} \in \Gamma\left(\mathbb{P}^{n}, \Omega_{\mathbb{P}^{n}}^{n-1}(\log (\mathcal{A}+\infty))(-\infty)\right) .
$$

In particular, $\gamma_{n-1}$ has log poles, so we can take the residue along components of $\mathcal{A}$. (The configuration $\mathcal{A}$ does not have normal crossings. The sheaf $\Omega_{\mathbb{P}^{n}}^{n-1}(\log (\mathcal{A}+\infty))$ is defined to be the subsheaf of $j_{*} \Omega_{\mathbb{P}^{n}-\mathcal{A}-\infty}^{n-1}$, where $j: \mathbb{P}^{n} \backslash(\mathcal{A} \cup \infty) \rightarrow \mathbb{P}^{n}$ is the open embedding, generated by forms without poles and the evident log forms with residue 1 along one hyperplane and $(-1)$ along another one. According to [1], the global sections of this naturally defined log sheaf compute de Rham cohomology.)

In the following, we adopt the sign convention $\operatorname{Res}_{t=0} \frac{d t}{t} \wedge \omega=\omega$. This yields

$$
\operatorname{Res}_{t=0} d\left(\frac{d t}{t} \wedge \omega\right)=-d\left(\operatorname{Res}_{t=0} \frac{d t}{t} \wedge \omega\right) .
$$

LEMMA 5.1. - We have the following residue formulae

$$
\operatorname{Res}_{v_{i}=0} \gamma_{n}=(-1)^{i} \gamma_{n-1} ; \quad 0 \leqslant i \leqslant n+1 .
$$

Proof. - One can either compute directly or argue indirectly as follows:

$$
\begin{aligned}
-d \operatorname{Res}_{v_{i}=0} \gamma_{n} & =\operatorname{Res}_{v_{i}=0} d \gamma_{n}=\operatorname{Res}_{v_{i}=0} \nu_{n+1} \\
& =(-1)^{i+1} \nu_{n}=-d\left((-1)^{i} \gamma_{n-1}\right) \neq 0 .
\end{aligned}
$$

$4^{\mathrm{e}}$ SÉRIE - TOME $36-2003-\mathrm{N}^{\circ} 3$ 
To conclude now, it suffices to show that the space of global sections (5.2) has dimension 1. To verify the dimension 1 property, let $\mathcal{A}^{\prime} \subset \mathcal{A}$ be defined by $V_{1} \cdots V_{n}=0$. Then $\mathcal{A}^{\prime}+\infty$ consists of $n+1$ hyperplanes in general position in $\mathbb{P}^{n}$, so

$$
\Omega_{\mathbb{P}^{n}}^{n-1}\left(\log \left(\mathcal{A}^{\prime}+\infty\right)\right)=\wedge^{n-1} \Omega_{\mathbb{P}^{n}}^{1}\left(\log \left(\mathcal{A}^{\prime}+\infty\right)\right) \cong \mathcal{O}_{\mathbb{P}^{n}}^{\oplus n}
$$

One looks at the evident residue

$$
\Omega_{\mathbb{P}^{n}}^{n-1}(\log (\mathcal{A}+\infty))(-\infty) \rightarrow \Omega_{\mathbb{P}^{n-1}}^{n-2}(\log (\mathcal{A}+\infty))(-\infty)
$$

along $V_{0}=0$.

Remark 5.2. - The computation of the lemma shows that $\gamma_{n-1}$ is the unique $(n-1)$ differential form in $\Gamma\left(\mathbb{P}^{n}, \Omega_{\mathbb{P}^{n}}^{n-1}(\log (\mathcal{A}+\infty))(-\infty)\right)$ with $d \gamma_{n-1}=\nu_{n}$. The configuration $\mathcal{A}$ being given, fixing $\infty$ fixes $\gamma_{n-1}$.

THEOREM 5.3. - The assignment $x \mapsto \operatorname{Tr}_{k(x) / k}(\gamma(x))$ gives an isomorphism for $n \geqslant 1$

$$
S H^{n}(k, n) \cong \Omega_{k}^{n-1} \text {. }
$$

Proof. - Let $X \subset Q_{k}^{n+1}$ be a curve in good position. For a zero-cycle $c$ in good position on $Q^{n}$ we write $\operatorname{Tr} \gamma_{n-1}(c) \in \Omega_{k}^{n-1}$ (absolute differentials) for the evident linear combination of traces from residue fields of closed points. We must show $\operatorname{Tr} \gamma_{n-1}(\partial X)=0$. Let $\bar{X}$ denote the closure of $X$ in $\mathbb{P}^{n}$. We consider $\left.\gamma_{n-1}\right|_{\bar{X}} \in \Gamma\left(\bar{X}, \Omega_{\bar{X} / \mathbb{Z}}^{n-1}(* D)\right)$, where $D$ is the pole set of $\left.\gamma_{n-1}\right|_{\bar{X}}$. The form $\gamma_{n-1}$ dies when restricted to $\infty$. Thus $D=\bigcup_{j=0}^{n} D_{j} \subset X$, with $D_{j}:=\partial_{j}\left(Q^{n}\right) \cap \bar{X}$. We define the residue along $D_{j}$ as follows. One has an exact sequence

$$
0 \rightarrow \Omega_{k}^{n} \otimes \mathcal{O}_{\bar{X}} \rightarrow \Omega_{\bar{X}}^{n}(\log D) \rightarrow \Omega_{k}^{n-1} \otimes \omega_{\bar{X}}(\log D) \rightarrow 0 .
$$

The residue map followed by the trace

$$
\omega_{\bar{X}}(\log D) \stackrel{\operatorname{Tr} \circ \sum \operatorname{Res}_{D_{j}}}{\longrightarrow} k
$$

yields, by the reciprocity formula, a vanishing residue map on global $n$-forms

$$
H^{0}\left(\bar{X}, \Omega_{\bar{X} / \mathbb{Z}}^{n}(\log D)\right) \rightarrow \Omega_{k}^{n-1} \bigotimes_{k} H^{0}(\bar{X}, \omega \bar{X}(\log D)) \stackrel{0}{\rightarrow} \Omega_{k}^{n-1} .
$$

On the other hand, the residue decomposes as

$$
H^{0}\left(\bar{X}, \Omega_{\bar{X} / \mathbb{Z}}^{n}(\log D)\right) \stackrel{\sum_{j} \operatorname{Res}_{D_{j}}}{\longrightarrow} \bigoplus_{j=0}^{n} \Omega_{D_{j}}^{n-1} \stackrel{\sum_{j} \operatorname{Tr}}{\longrightarrow} \Omega_{k}^{n-1} .
$$

By Lemma 5.1, $\gamma_{n-1}\left(D_{j}\right)=\left(\operatorname{Res}_{v_{j}=0} \gamma_{n}\right)\left(D_{j}\right)$. The desired vanishing follows.

We now have

$$
\Omega_{k}^{n-1} \stackrel{\phi}{\rightarrow} S H^{n}(k, n) \stackrel{\gamma_{n-1}}{\longrightarrow} \Omega_{k}^{n-1} .
$$

It suffices to check the composition is multiplication by $(-1)^{n+1}$. Given $b_{1}, \ldots, b_{n-1} \in k$ with $c:=\sum b_{i}-1 \neq 0$, the composition is computed to be (use (5.1) and Proposition 4.3) 


$$
\begin{aligned}
& a \cdot d \log \left(b_{1}\right) \wedge \cdots \wedge d \log \left(b_{n-1}\right) \mapsto \\
& \quad-a \sum(-1)^{i} d \log \left(\frac{b_{1}}{a c}\right) \wedge \cdots \wedge d \overline{\log \left(\frac{b_{i}}{a c}\right) \wedge \cdots \wedge d \log \left(\frac{-1}{a c}\right) .}
\end{aligned}
$$

Expanding the term on the right yields

$$
-a \sum(-1)^{i} d \log \left(\frac{b_{1}}{c}\right) \wedge \cdots \wedge d \widehat{\log \left(\frac{b_{i}}{c}\right)} \wedge \cdots \wedge d \log \left(\frac{-1}{c}\right)-a \cdot d \log (a) \wedge(\ldots),
$$

and it is easy to check that the terms involving $d \log (a)$ cancel. In this way, one reduces to the case $a=1$. Here

$$
\begin{gathered}
-\sum(-1)^{i} d \log \left(\frac{b_{1}}{c}\right) \wedge \cdots \wedge d \widehat{\log \left(\frac{b_{i}}{c}\right)} \wedge \cdots \wedge d \log \left(\frac{-1}{c}\right) \\
=(-1)^{n+1} \frac{d b_{1}}{b_{1}} \wedge \cdots \wedge \frac{d b_{n-1}}{b_{n-1}}+\frac{d c}{c} \wedge(\ldots) .
\end{gathered}
$$

Again the terms involving $d \log (c)$ cancel formally, completing the proof.

Challenge 5.4.-Finally, as a challenge we remark that the Kähler differentials have operations (exterior derivative, wedge product,...) which are not evident on the cycles $\mathcal{S Z}$. For example, one can show that the map

$$
\nabla\left(x_{0}, \ldots, x_{n}\right)=\left(x_{0},-\frac{x_{1} x_{0}}{1-x_{0}}, \ldots,-\frac{x_{n} x_{0}}{1-x_{0}},-\frac{x_{0}}{1-x_{0}}\right)
$$

satisfies

$$
\gamma_{n}(\nabla(x))=(-1)^{n} d \gamma_{n-1}(x)
$$

and hence induces the exterior derivative on the 0 -cycles. The map is not uniquely determined by this property, and this particular map does not preserve good position for cycles of dimension $>0$. Can one find a geometric correspondence on the complex $\mathcal{S Z}{ }^{\bullet}$ which induces $d$ on the 0 -cycles? What about the pairings $(a, b) \mapsto a \wedge b$ or $(a, b) \mapsto a \wedge d b$ ?

\section{Specialization of forms}

In this section we consider again the specialization of differential forms as in Section 1. Recall $f\left(U_{0}, \ldots, U_{n+1}\right)$ is homogeneous of degree $n+2, u_{i}=U_{i} / U_{0}$, and $v_{i}=t^{r_{i}} u_{i} . N$ is minimal such that $t^{N} f\left(1, t^{-r_{1}} v_{1}, \ldots, t^{-r_{n+1}} v_{n+1}\right)$ is integral in $t$, and we assume $s=N-\sum r_{i}>0$. We have forms

$$
\omega_{f}:=\frac{d\left(t^{-r_{1}} v_{1}\right) \wedge \cdots \wedge d\left(t^{-r_{n+1}} v_{n+1}\right)}{f\left(1, t^{-r_{1}} v_{1}, \ldots, t^{-r_{n+1}} v_{n+1}\right)}=t^{s} \nu_{f}+s t^{s-1} d t \wedge \gamma_{f}
$$

and $\left.\nu_{f}\right|_{t=0}=\left.d \gamma_{f}\right|_{t=0}$.

We have already mentioned the case

$$
f=\Delta_{n+1}=\left(t^{-1} v_{1}\right) \cdots\left(t^{-1} v_{n+1}\right)\left(1-t^{-1} v_{1}-\cdots-t^{-1} v_{n+1}\right) .
$$

The forms $\nu_{\Delta_{n+1}}$ and $\gamma_{\Delta_{n+1}}$ are given in (1.8). As before, we write $\nu_{n+1}:=\left.\nu_{\Delta_{n+1}}\right|_{t=0}$; $\gamma_{n}:=\left.\gamma_{\Delta_{n+1}}\right|_{t=0}$. The differential form $\nu_{n+1}$ plays an interesting rôle in the computation of de 
Rham cohomology of the complement of hyperplane configurations. Let $\mathcal{A}_{t}$ be the configuration in $\mathbb{P}^{n}$ of $(n+1)$ hyperplanes in general position with affine equation $u_{0} u_{1} \cdots u_{n}=0$, $u_{0}+u_{1}+\cdots+u_{n}=t \neq 0$. Then $H^{n}\left(\mathbb{P}^{n} \backslash \mathcal{A}_{t}\right)=H^{0}\left(\mathbb{P}^{n}, \Omega_{\mathbb{P}^{n}}^{n}\left(\log \mathcal{A}_{t}\right)\right)$ is a pure Tate structure generated by $\nu_{\Delta n+1}$. Now make $t$ tend to 0 and consider the degenerate configuration $\mathcal{A}_{0}$ with affine equation $u_{0} u_{1} \cdots u_{n}=0, u_{0}+u_{1}+\cdots+u_{n}=0$. Exactness of $\nu_{n+1}=d \gamma_{n}$ in the case (1.7) follows from Aomoto's theory [1,4]. Due to the special shape of the configurations $\mathcal{A}_{t}$ considered, however, more is true. We have fixed $\infty$, and the differential form $\gamma_{n}$ lies in $H^{0}\left(\mathbb{P}^{n+1}, \Omega^{n}\left(\log \left(\mathcal{A}_{0}+\infty\right)\right)(-\infty)\right)$. That is, it has log poles along all components of $\mathcal{A}_{0}$, and it vanishes as a differential form at $\infty$ (see Lemma 5.1). This $k$-vector space of differential forms is 1-dimensional. In other words, the differential form $\gamma_{n}$ is uniquely defined by the vanishing condition at $\infty$ and the secondary data $d \gamma_{n}=\nu_{n+1}$ (see Remark 5.2). Do such primitives for more general degenerating configurations admit an interpretation identifying 0 -cycles with spaces of differential forms?

Here is another example of specialization and 0-cycles. Define

$$
\begin{gathered}
f\left(1, u_{1}, u_{2}\right)=u_{1}^{2}-u_{2}^{3}-a u_{2}-b ; \\
v_{1}=t^{3} u_{1}, \quad v_{2}=t^{2} u_{2} .
\end{gathered}
$$

One computes

$$
\nu=\frac{d v_{1} \wedge d v_{2}}{v_{1}^{2}-v_{2}^{3}-a t^{4} v_{2}-b t^{6}} ; \quad \gamma=\frac{2 v_{2} d v_{1}-3 v_{1} d v_{2}}{v_{1}^{2}-v_{2}^{3}-a t^{4} v_{2}-b t^{6}}
$$

One checks that $\operatorname{Res}\left(\left.\gamma\right|_{t=0}\right)=v_{2} / v_{1}$ and

$$
d\left(v_{2} / v_{1}\right)=-d v_{2} / 2=d \operatorname{Res}\left(\left.\nu\right|_{t=0}\right) \quad \text { on } v_{1}^{2}-v_{2}^{3}=0 .
$$

The assignment $x \mapsto \operatorname{Tr}_{k(x) / k}\left(v_{2} / v_{1}\right)(x)$ identifies the jacobian of the special fibre $v_{1}^{2}-v_{2}^{3}=0$ with $\mathbb{G}_{a}(k)=k$.

A final example of specialization, which we understand less well, though it was an inspiration for this article, concerns the hyperbolic motives of Goncharov [6]. The matrix coefficients of his theory (in the sense of [2]) are the objects $H^{2 n-1}\left(\mathbb{P}^{2 n-1} \backslash Q, M \backslash Q \cap M\right)$. Here $Q \subset \mathbb{P}^{2 n-1}$ is a smooth quadric and $M$ is a simplex (union of $2 n$ hyperplanes in general position). The subschemes $Q$ and $M$ are taken in general position with respect to each other. The notation is intended to suggest a sort of abstract relative cohomology group. In de Rham cohomology, the non-trivial class in $H_{D R}^{2 n-1}\left(\mathbb{P}^{2 n-1} \backslash Q\right)$ is represented by

$$
\omega=\frac{d u_{1} \wedge \cdots \wedge d u_{2 n-1}}{\left(u_{1}^{2}+\cdots+u_{2 n-1}^{2}-1\right)^{n}} .
$$

Substituting $u_{i}=v_{i} t$, we get

$$
\omega=\frac{t^{2 n-1} d v_{1} \wedge \cdots \wedge d v_{2 n-1}+t^{2 n-2} d t \wedge \sum(-1)^{i} v_{i} d v_{1} \wedge \cdots \widehat{d v_{i}} \cdots \wedge d v_{2 n-1}}{\left(t^{2}\left(v_{1}^{2}+\cdots+v_{2 n-1}^{2}\right)-1\right)^{n}}
$$

We get

$$
\left.\nu\right|_{t=0}= \pm d v_{1} \wedge \cdots \wedge d v_{2 n-1}
$$


and

$$
\left.\gamma\right|_{t=0}=\frac{1}{2 n-1} \sum(-1)^{i} v_{i} d v_{1} \wedge \cdots \widehat{d v_{i}} \cdots \wedge d v_{2 n-1} .
$$

Let $\Delta_{M} \in H_{2 n-1}\left(\mathbb{P}^{2 n-1}, M ; \mathbb{Z}\right)$ be a generator. The hyperbolic volume

$$
\int_{\Delta_{M}} \omega
$$

is the real period [6, Section 4.1] of the Hodge structure associated to $H^{2 n-1}\left(\mathbb{P}^{2 n-1} \backslash Q\right)$. Goncharov remarks (op. cit., Question 6.4 and Theorem 6.5) that this volume degenerates to the euclidean volume as $t \rightarrow 0$. (More precisely, from (6.7), we see that as a relative form, $d v_{1} \wedge \cdots \wedge d v_{2 n-1}=\lim _{t \rightarrow 0} t^{1-2 n} \omega$.) He asks for an interpretation of the degenerated volume in terms of some sort of motive over $k[t] /\left(t^{2}\right)$.

In the Goncharov picture we can view $Q$ as fixed and degenerate $M$. Suppose

$$
M: L_{0} L_{1} \cdots L_{2 n-1}=0
$$

where

$$
L_{i}=L_{i}\left(v_{1}, \ldots, v_{2 n-1}\right)=L_{i}\left(u_{1} / t, \ldots, u_{2 n-1} / t\right) .
$$

Assuming the $L_{i}$ are general, clearing denominators and passing to the limit $t \rightarrow 0$ yields a degenerate simplex $M_{0}$ consisting of $2 n$ hyperplanes meeting at the point $v=0$. This limiting configuration leads to the Chow groups $S H^{*}(k, 2 n-1)$, but we do not see how to relate $\left.\gamma\right|_{t=0}$ to cycles.

\section{Cycle groups of divisors}

We compute $S H^{1}(k, n)$ for $n \geqslant 1$.

Let $\mathcal{O}_{Q^{n}, 0}$ be the local ring at $(0, \ldots, 0) \in Q^{n}$. Let

$$
I^{n} \subset J^{n} \subset \mathcal{O}_{Q^{n}, 0}
$$

be the ideals of all (resp. all but the last) face.

LEMMA 7.1.-

$$
S H^{1}(k, n) \cong\left(1+I^{n}\right)^{\times} / \partial_{n+1}\left(1+J^{n+1}\right)^{\times} .
$$

Proof. - Let $D \subset Q^{n}$ be an effective divisor. Let

$$
f \in k_{n}:=k\left[t_{0}, \ldots, t_{n}\right] /\left(\sum t_{i}\right)
$$

be a defining equation for $D$. Then $D$ meets faces properly in our sense if and only if $f \in \mathcal{O}_{Q^{n}, 0}^{\times}$. Conversely, any $f \in \mathcal{O}_{Q^{n}, 0}^{\times}$defines a (not necessarily effective) divisor on $Q^{n}$ meeting faces properly. Further

$$
(f) \cdot Q_{i}^{n-1}=0 \quad \Leftrightarrow \quad f \in k^{\times} \cdot\left(1+t_{i} \mathcal{O}_{Q^{n}, 0}\right) .
$$

By general simplicial considerations, the $S H^{1}(k, n)$ is given by divisors meeting all faces trivially, modulo the restriction to the last face of divisors on $Q^{n+1}$ meeting all but the last 
face trivially. Scaling the $f$ above to remove the factor $k^{\times}$, this is precisely the assertion of the lemma.

Proposition 7.2. - We have $S H^{1}(k, 1)=k$, and $S H^{1}(k, n)=(0)$ for $n \geqslant 2$.

Proof. - The first assertion is part of Theorem 5.3. The exact sequence

$$
0 \rightarrow I^{n+1} \rightarrow J^{n+1} \stackrel{\partial_{n+1}}{\longrightarrow} I^{n} \rightarrow 0
$$

yields the surjectivity $1+J^{n+1} \rightarrow 1+I^{n}$. We observe that when $n=1$, we have $I^{1}=\left(t_{0}\right)$ but $\partial_{2}\left(J^{2}\right)=\left(t_{0}^{2}\right)$.

\section{Acknowledgements}

The authors wish to thank A. Goncharov and S. Lichtenbaum for several very inspiring conversations, also the referee for very careful reading and helpful remarks.

\section{REFERENCES}

[1] Аомото K., Un théorème du type de Matsushima-Murakami concernant l'intégrale des fonctions multiformes, J. Math. Pures Appl. 52 (1973) 1-11.

[2] Beilinson A., Goncharov A., Schechtman V., Varchenko A., Aomoto dilogarithms, mixed Hodge structures, and motivic cohomology of a pair of triangles in the plane, in: The Grothendieck Festschrift, Birkhäuser, 1990, pp. 131-172.

[3] BLOCH S., Algebraic cycles and higher K-theory, Adv. Math. 61 (3) (1986) 267-304.

[4] BRIESKorn E., Sur les groupes de tresses, in: Sém. Bourbaki 401, in: Lect. Notes in Math., Vol. 317, Springer, Berlin, 1973, pp. 21-44.

[5] Cathelineau J.-L., Remarques sur les différentielles des polylogarithmes uniformes, Ann. Inst. Fourier, Grenoble 46 (5) (1996) 1327-1347.

[6] GonchaRov A., Volumes of hyperbolic manifolds and mixed Tate motives, JAMS 12 (2) (1999) 569618.

[7] Nesterenko Yu., Suslin A., Homology of the general linear group over a local ring, and Milnor's K-theory, Izv. Akad. Nauk SSSR Ser. Math. 53 (1) (1989) 121-146; translation in: Math. USSRIzv. 34 (1) (1990) 121-145.

[8] Totaro B., Milnor $K$-theory is the simplest part of algebraic $K$-theory, $K$-Theory 6 (2) (1992) 177 189.

[9] Voevodsky V., Motivic cohomology groups are isomorphic to higher Chow groups in any characteristic, Int. Math. Res. Not. 7 (7) (2002) 351-355.

Spencer BLOCH

Department of Mathematics,

University of Chicago,

Chicago, IL 60637, USA

E-mail: bloch@math.uchicago.edu

Hélène ESNAULT

Universität Essen,

FB6, Mathematik,

45117 Essen, Germany

E-mail: esnault@uni-essen.de 